- Odontogenic infections can have severe complications.

- The diagnosis of necrotising fasciitis requires a high index of suspicion.

- Long term sequelae can be minimised with prompt and appropriate management.

\title{
Necrotising fasciitis of the submandibular region; a complication of odontogenic origin
}

\author{
J. N. Farrier, ${ }_{1}^{1}$ M. A. Kittur ${ }^{2}$ and A. W. Sugar ${ }^{3}$
}

\begin{abstract}
Inadequate treatment or neglect of odontogenic infections can have serious consequences. The potential for spreading through fascial planes and intracranially can cause compromise of the airway and cavernous sinus thrombosis respectively. On rare occasions this can lead to a rapidly progressing necrotising fasciitis, with destruction of soft tissue, making reconstruction difficult. Antibiotic administration without removal of the cause is inadequate and can complicate subsequent management. We report the presentation and successful management of a 13-year-old boy, who developed necrotising fasciitis in the submandibular region as a result of inadequate initial treatment of a carious, lower molar resulting in significant skin and soft tissue loss.
\end{abstract}

\section{INTRODUCTION}

Head and neck infections in children are common. The multiple anatomical structures present within this area result in significant clinical and bacteriological variability. Children with facial swelling, pain and fever often initially present to paediatricians, ophthalmologists, and infectious disease physicians before being seen by an oral and maxillofacial surgeon. ${ }^{1}$ Carious teeth are a common source of infection in the head and neck region. ${ }^{2}$ Serious complications have been reported from odontogenic spread of infection. These include airway compromise, cavernous sinus thrombosis, blindness and even death. ${ }^{3,4}$ Most of these complications can be prevented by

Specialist Registrar, ${ }^{2}$ Senior House Officer, ${ }^{3}$ Consultant Maxillofacial Surgeon, Department of Oral \&t Maxillofacial Surgery, Morriston Hospital, Swansea, SA6 6NL

*Correspondence to Mr J. Farrier

Email:sarah-jerry@farrier27.wanadoo.co.uk

\section{Refereed Paper}

Accepted 23 August 2006

DOI: 10.1038/bdj.2007.425

${ }^{\circ}$ British Dental Journal 2007; 202: 607-609 timely intervention, appropriate antibiotic therapy, extraction of the offending teeth and incision and drainage of any collection.

A rare complication is necrosis with subsequent loss of facial skin. Necrotising fasciitis (NF) is a rare but lifethreatening polymicrobial soft tissue infection characterised by progressive, and usually rapid, necrosis of the subcutaneous tissues and fascial planes, resulting in skin loss and systemic toxicity. ${ }^{5}$ The condition is relatively common in the extremities, abdominal wall and perineum, but is rarely seen in the head and neck. Because of the fulminant course of NF, early diagnosis is imperative. Broad-spectrum antibiotics, aggressive surgical treatment and supportive therapy are the mainstay of successful treatment. ${ }^{5}$

Necrotising fasciitis is a clinical diagnosis. Initially, the skin is smooth, tense and shiny with no sharp demarcation, and progresses to develop a dusky discolouration with poorly defined borders, with localised necrosis of skin, which if untreated, will progress to frank cutaneous gangrene. There is associated pain, swelling, erythema, and oedema, which can be mistaken for cellulitis or erysipelas. Crepitus is common due to the presence of gas forming organisms. Unfortunately, the disease is often diagnosed late in its course, resulting in high mortality, therefore a high index of suspicion is obligatory. ${ }^{6,7}$

This report highlights the management of an otherwise fit and healthy 13-year-old boy, with severe necrotising fasciitis of odontogenic origin.

\section{CASE REPORT}

A 13-year-old boy was referred to the Maxillofacial unit in Morriston Hospital Swansea by his general dental practitioner with a two week history of a progressively increasing swelling in the left submandibular region.

The initial presentation to the dentist was for dental pain arising from the lower left quadrant which was followed by the development of a swelling. He was prescribed oral penicillin $250 \mathrm{mg}$, 


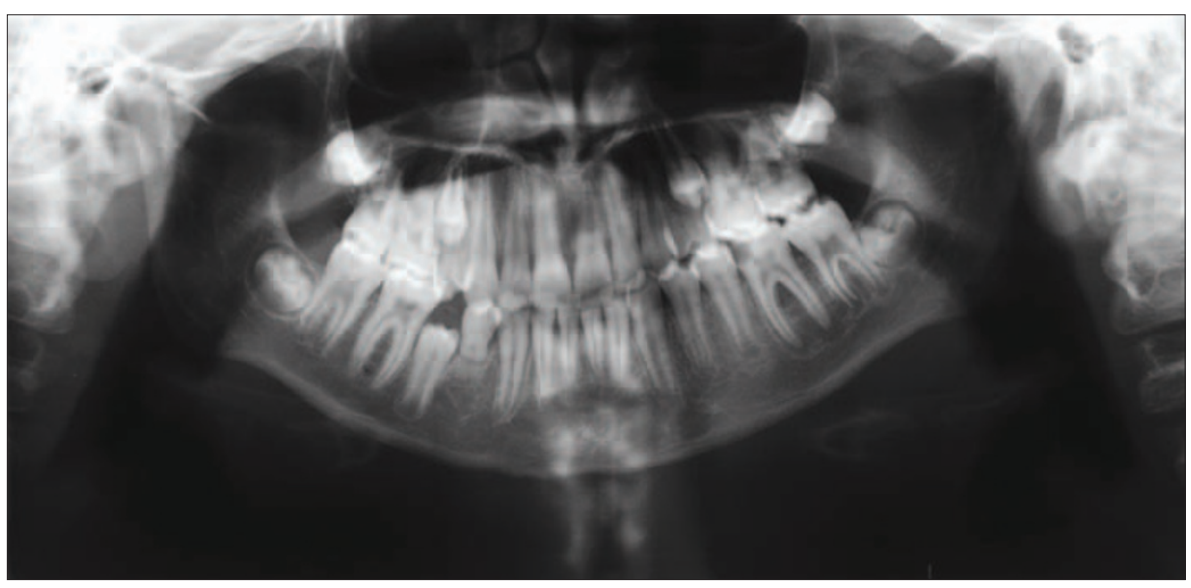

Fig. 1 Radiographic appearance of carious LL6; causative factor in necrotising fasciitis.

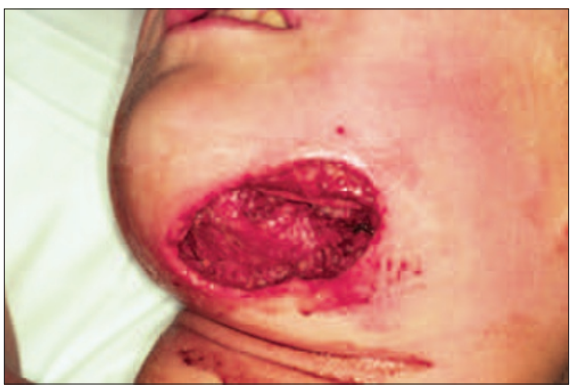

Fig. 2 Clinical appearance following debridement of necrotic tissue.

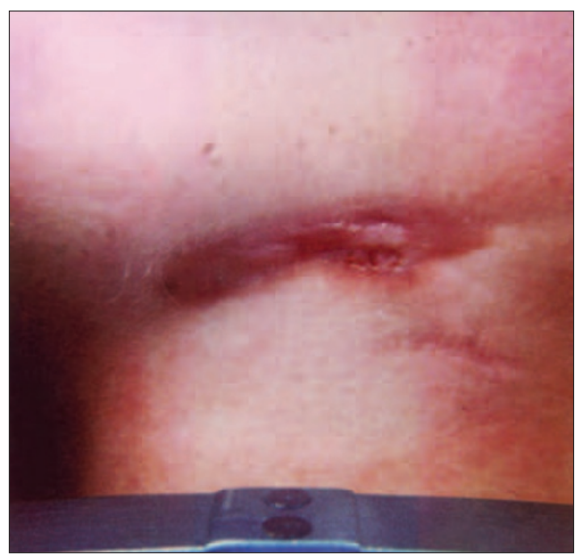

Fig. 3 Appearance of scar, three weeks post-operatively.

six hourly. No attempt was made to eliminate the cause and the swelling continued to increase in size.

On presentation to the maxillofacial team the patient appeared dehydrated and was febrile with a temperature of $38.6^{\circ} \mathrm{C}$. He had marked trismus (maximal inter-incisal opening of $6 \mathrm{~mm}$ ) and complained of difficulty in swallowing. There was no airway compromise. A large brawny hard swelling was evident on the left side of the face involving the buccal and sub-mandibular spaces, which crossed the midline into the contralateral submandibular space. Skin overlying the swelling was erythematous, inflamed and taut, with a small central area of fluctuance. Intraoral and radiological examination revealed the lower left first molar to have a large mesio-occlusal carious lesion involving the pulp, with evidence of small periapical radiolucency (Fig. 1). Routine haematological investigations showed a marked rise in WBC (17.2 109/1) with a predominant neutrophilia suggesting acute bacterial infection, and the patient was started on a course of intravenous Co-Amoxiclav (1.2 g eight hourly) and Metronidazole (500 mg eight hourly). Due to the potential risk of airway obstruction, the patient was taken to theatre within the hour for incision and drainage of the abscess and extraction of the offending tooth.

Copious amounts (30-40 ml) of foul smelling pus were drained and samples sent for microbiology, culture and sensitivity, Gram's and Ziehl-Nielsen staining. Locules in the abscess cavity were opened by blunt dissection and all necrotic tissue excised. Removal of the necrotic skin and muscle left a sizeable defect in the skin measuring $3 \times 5 \mathrm{~cm}$ exposing the lower border of the mandible (Fig. 2). The cavity was thoroughly cleaned with copious amounts of warm saline and loosely packed with Betadine soaked gauze. The initial dressing remained in situ for 48 hours before being changed daily for a further two days, prior to the placement of Silastic foam. Regular swabs of the cavity were sent for micro culture and sensitivity. Antibiotic treatment was discontinued after three negative swabs had been obtained (total duration of antibiotics was eight days).

Microbiological analysis of the pus showed the organisms to be a mixed flora of Streptococcus viridans, Streptococcus $C$ and anaerobes sensitive to Co-amoxiclav and Metronidazole. Histo-pathology was reported as necrotic muscle tissue.

After five days of dressing with silastic foam, the wound was allowed to heal by secondary intention. One week after the procedure the cavity had reduced in size $2 \times 3 \mathrm{~cm}$ and the exposed bone was covered with healthy granulation tissue. Three weeks post-operatively the cavity had completely closed leaving only a broad linear scar (Fig. 3).

The diagnosis of necrotising fasciitis was made on the basis of the amount of soft tissue loss, the presence of intensely offensive pus, and the type of organisms cultured.

\section{DISCUSSION}

The term 'necrotising fasciitis' was first coined by Wilson in 1952, most commonly occurring in the perineum, lower limbs or abdominal wall following surgery or trauma, particularly in individuals with underlying systemic diseases such as diabetes mellitus, arteriosclerosis, chronic renal failure or malnutrition. ${ }^{8-10}$ NF may affect patients of all ages, with no sex or race predilection. ${ }^{11}$ Clinical signs and symptoms are nonspecific. The skin is red-hot, smooth, tense and tender without sharp demarcation between the involved and normal skin. Crepitation may be elicited. As the disease progresses, the skin becomes dusky and small blisters appear followed by frank gangrene. Skin necrosis is secondary to thrombosis of the nutrient vessels passing through necrotic fascia. ${ }^{12}$ The patient is systemically unwell with leucocytosis, and regional lymphadenopathy. ${ }^{13}$

Prompt and successful management of NF is universally agreed to be aggressive surgical intervention, intravenous antibiotics and supportive therapy. ${ }^{2-19}$ It is of paramount importance to explore and drain all affected areas. Early surgical intervention will minimise loss of the covering skin and avoid cosmetic disfiguring. ${ }^{14}$

Wound dressings soaked in normal saline or Betadine as topical treatment after debridement have been used but we believe that the frequency of dressing is far more important than the solution used. Broad-spectrum antibiotics covering both aerobic and anaerobic organisms should be initiated and changed 
according to the sensitivity patterns. Antibiotic therapy alone is considered insufficient for the management of NF without surgical intervention. ${ }^{15}$ The local and systemic complications of NF are numerous and include extension to the cervical viscera and mediastinum, vessel erosion and functional and cosmetic disfiguring. ${ }^{16}$ Death from necrotising fasciitis is usually due to overwhelming sepsis, respiratory, renal, or multi-organ failure. ${ }^{17}$ The mortality rate ranges between $8 \%$ and $73 \% .{ }^{9}$ The principal factors contributing to the high mortality are delayed diagnosis and treatment, extent of the disease, old age and associated systemic illnesses. ${ }^{18,19}$

The presented case emphasises the need for early diagnosis, proper aggressive surgical intervention and intravenous antibiotic therapy of this serious, life threatening infection. The condition must be recognised as early as possible to prevent unnecessary morbidity and mortality. It emphasises the need to create awareness among dental practitioners the importance of treating odontogenic infections as soon as possible and to initiate rapid referral to the nearest maxillofacial unit as necessary.

1. Dodson T B, Kaban L B, Perrott D H. Paediatric maxillofacial infections: a retrospective study of 113 patients. J Oral Maxillofac Surg 1989; 47: 327-330.

2. Zeitoun I M, Dhanarajani P J. Cervical cellulites and mediastinitis caused by odontogenic infections: report of two cases and review of literature. J Ora Maxillofac Surg 1995; 53: 203-208.

3. Green A W, Flower E A, New N E. Mortality associated with Odontogenic infection! Br Dent J 2001; 190: 529-530.

4. Krishnan V, Johnson J V, Helfrick J F. Management of maxillofacial infections: review of 50 cases. J Oral Maxillofac Surg 1993; 51: 868-873.

5. Scaturi P Jr, Dodson T B. Epidemiologic review of paediatric and adult maxillofacial infections in hospitalised patients. Oral Surg Oral Med Oral Pathol 1996; 81: 270-274.

6. Dodson T B, Kaban L B. Special considerations for paediatric emergency patient. Emerg Med Clin N America 2000; 18: 539-548.

7. Strachan D D, Williams FA, Bacon W J. Diagnosis and treatment of paediatric maxillofacial infections. Gen Dent 1998; 46: 180-182.

8. Balcerak R J, Sisto J M, Bosack R C. Cervicofacial necrotizing fasciitis: report of three cases and literature review. J Oral Maxillofac Surg 1988: 46: 450-459.

9. Reed J M, Anand V K. Odontogenic cervical necrotizing fasciitis with intrathoracic extension. Otolaryngol Head Neck Surg 1992; 107: 596-600.

10. Valko P C, Barrett S M, Campbell J P. Odontogenic cervical necrotizing fasciitis. Ann Emerg Med 1990: 15: 568-571.

11. Kaddour H S, Smelt G J C. Necrotizing fasciitis of the neck. J Laryngol Otol 1992; 106: 1008-1010.

12. Maqbool M, Ahmad R, Ahmed A et al. Necrotizing fasciitis in the head and neck. Br J Plast Surg 1992; 45: 481-483

13. Walters R. A fatal case of necrotizing fasciitis of the eyelid. Br J Ophthalmol 1988; 72: 428-431.

14. Stamenkovic I, Lew P D. Early recognition of potentially fatal necrotizing fasciitis: the use of frozen section biopsy. N Eng J Med 1984; 310: 1689-1693.

15. Kronish J W, McLeish W M. Eyelid necrosis and periorbital necrotizing fasciitis. Report of a case and review of the literature. Ophthalmol 1991; 98: 92-98.

16. Lalwani A K, Kaplan M J. Mediastinal and thoracic complications of necrotizing fasciitis of the head and neck. Head Neck 1991; 13: 531-539.

17. Kiely P D, Gilvarry A. Periorbital necrotizing fasciitis: trivial facial injury resulting in cardiac arrest. Br J Clin Prac 1993; 47: 169-170.

18. Gaukroger M C. Cervicofacial necrotizing fasciitis. Br J Oral Maxillofac Surg 1992; 30: 111-114.

19. Rapoport Y, Himelfarb M Z, Zikk D et al. Cervical necrotizing fasciitis of odontogenic origin. Oral Surg Oral Med Oral Pathol 1991; 72: 15-18. 